\title{
A novel patient-reported outcome instrument assessing the symptoms of paroxysmal nocturnal hemoglobinuria, the PNH-SQ
}

\author{
R. Paola Daly ${ }^{1}$, Jessica J. Jalbert ${ }^{2 *}$, Shannon Keith ${ }^{1}$, Tara Symonds ${ }^{3}$ and Jamile Shammo ${ }^{4}$
}

\begin{abstract}
Background: Patient-reported outcome measures (PROs) used to measure symptoms of patients with paroxysmal nocturnal hemoglobinuria (PNH) in trials do not measure PNH symptoms comprehensively and do not assess daily fluctuations in symptoms. Following a literature review and consultation with a PNH expert, we drafted the PNH Symptom Questionnaire (PNH-SQ) and a patient-centric conceptual model of PNH symptoms and impacts. We then interviewed 15 patients with PNH to assess comprehensiveness of symptom capture from the patient perspective and to cognitively debrief the PNH-SQ. Patient interview data were also used to finalize the PNH conceptual model.

Results: Participants mentioned 27 signs or symptoms of PNH spontaneously or after being probed; $93 \%$ reported experiencing $\geq 1$ PNH symptom. Concept saturation was reached for all PNH symptoms. Further, interviews confirmed the instrument captured the most common PNH symptoms, including fatigue (87\%), abdominal pain (60\%), and difficulty swallowing (47\%), with fatigue ranked as the most bothersome symptom. The interviews demonstrated that participants understood the items of the PNH-SQ (90-100\%); considered the symptoms relevant (>50->90\%); the recall period appropriate (> 80-100\%); and the response options suitable (>80-100\%). Participants also suggested changes regarding item redundancy and relevance; this feedback was used to finalize the instrument.

Conclusions: The finalized PNH-SQ assesses the presence and severity of 10 symptoms-abdominal pain, chest discomfort, difficulty sleeping, difficulty swallowing, difficulty thinking clearly, fatigue, headache, muscle weakness, pain in the legs or back, and shortness of breath-over $24 \mathrm{~h}$. The PNH-SQ is a content-valid questionnaire suitable for assessing daily symptom presence and severity in PNH clinical trials.
\end{abstract}

Keywords: PNH, PRO, QoL, Symptom

\section{Background}

Paroxysmal nocturnal hemoglobinuria $(\mathrm{PNH})$ is a rare, acquired, life-threatening disease of the blood caused by a chronic dysregulation of the complement system [1] and is characterized by hemolytic anemia, thrombosis, and impaired bone marrow function $[2,3]$. The prevalence of

\footnotetext{
*Correspondence: jessica.jalbert@regeneron.com

${ }^{2}$ Regeneron Pharmaceuticals, Inc., 777 Old Saw Mill River Rd, Tarrytown, NY 10591, USA

Full list of author information is available at the end of the article
}

PNH is estimated to be between 10 and 15 per million [4, 5], with a mean age of diagnosis between 30 and 45 years [6]. Patients with PNH experience a high symptom burden that impacts and significantly reduces quality of life $[3,5,7]$. In the International PNH Registry, among the 856 patients for whom symptom data were available, the most commonly reported symptoms were dyspnea (64\%), headache $(63 \%)$, and fatigue (80\%) [7]. Patients experiencing symptoms of PNH in the previous 6 months had significantly lower quality of life scores, as measured by the European Organisation for Research and Treatment 
of Cancer Quality of Life of Cancer Patients (EORTC QLQ-C30), than patients not experiencing symptoms [7]. In addition, in a subset of 506 participants aged 18-59, $17.4 \%$ reported that their $\mathrm{PNH}$ was the reason they were not working or working part-time instead of full-time [7]. In a study consisting of concept elicitation interviews with 29 patients with $\mathrm{PNH}, 97 \%$ of participants reported experiencing fatigue, $76 \%$ experienced headache, $66 \%$ experienced dyspnea, and 59\% experienced abdominal pain [8]. Over one-half of patients reported symptom severity as moderate to severe and some patients experienced certain symptoms with high frequency; 52\% of those with fatigue and $41 \%$ of those with headaches reported that their symptoms occurred frequently or almost constantly [8]. The percent of patients who were unemployed due to $\mathrm{PNH}$ was similar to that reported in the International PNH Registry (17\%) [8].

Given that most patients with PNH experience symptoms that have a substantial impact on quality of life and ability to perform daily activities, symptoms should be carefully measured and tracked in trials evaluating new treatment options for $\mathrm{PNH}$. We conducted a literature review and no fit-for-purpose patient-reported outcome (PRO) measure existed to assess symptoms of PNH in clinical trials. While the EORTC QLC-C30 and Functional Assessment of Chronic Illness Therapy-Fatigue (FACIT-Fatigue) have been used to support labeling for $\mathrm{PNH}$ treatments, these measures were not developed specifically for patients with PNH. The EORTC QLQC30 has significant gaps in symptom coverage and measures symptoms that are not relevant to patients with
PNH (e.g. nausea, vomiting, constipation) [6, 8]. Moreover, both the EORTC-QLQ-C30 and the FACIT-Fatigue assess symptoms over the past week and interviews with patients with PNH suggest that symptom frequency may vary [8].

The objective of this study was to gain a better understanding of the patient experience related to $\mathrm{PNH}$ symptoms and to develop a content-valid questionnaire-the $\mathrm{PNH}$ Symptom Questionnaire (PNH-SQ) - for use in clinical trials to assess the presence, severity, and day-today variations in $\mathrm{PNH}$-specific symptoms.

\section{Methods}

The development of the PNH-SQ was conducted in accordance with the Food and Drug Administration (FDA)'s guidance on PRO measure development [9]. The content and design of the PNH-SQ was informed by a review of the empirical literature, review of the content of PRO measures used in registrational trials to assess symptoms of PNH (i.e., the FACIT-Fatigue and the EORTC-QLQ-C30), and, in October 2018, through a discussion with a hematologist with over 20 years of experience treating patients with $\mathrm{PNH}$. The literature search was conducted in OVID across three databases (Medline, PsychInfo, and Embase) using the terms described in Fig. 1 and between the time period 2006-2018. This time period was chosen so that measure development articles published from the issuance of the draft FDA Guidance on PRO development [10] to the year in which the search was conducted would be included. From this search, four qualitative research papers were identified and

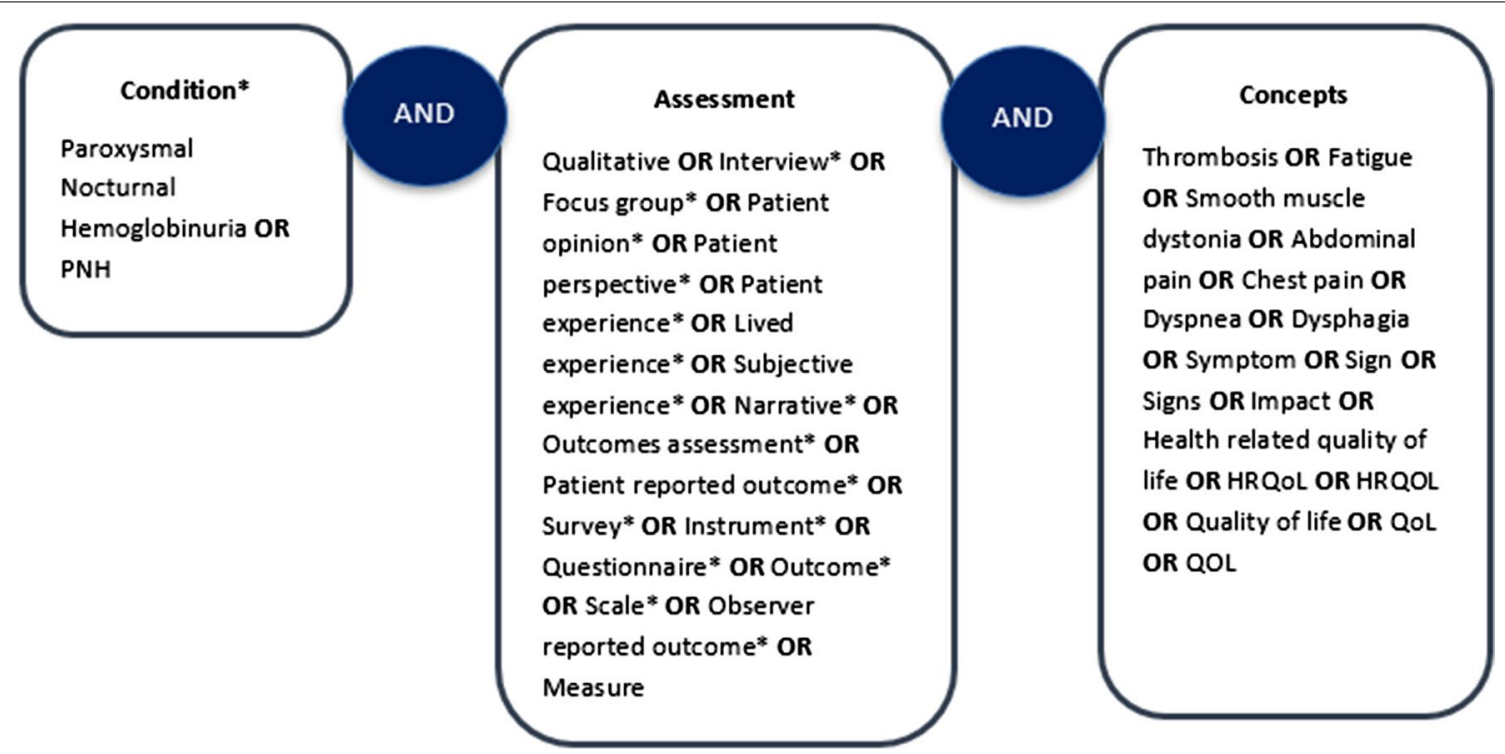

Fig. 1 Literature review search terms 
reviewed [7, 11-13]. Data on symptoms were extracted from each study. The results of the literature review were coupled with findings from the PRO review and clinician discussions to inform the development of a preliminary conceptual model of $\mathrm{PNH}$ and the first draft of the questionnaire. A translatability assessment was also conducted by a global translation company, Transperfect, on the PNH-SQ in 14 other languages including Czech, German, Italian, Korean, Polish, Thai, as well as UK and South African English. For this review, a linguistic validation expert reviewed the English version of the PNH-SQ to identify any text or concepts that could be difficult to translate. We then conducted hybrid patient interviews which consisted of concept elicitation (CE) and cognitive debriefing (CD). Patient feedback and discussions with a physician with experience treating patients with $\mathrm{PNH}$ (author JS) were then used to finalize the instrument.

\section{Concept selection and PNH-SQ construction}

The selection of key PNH symptoms for inclusion in the PNH-SQ was based on findings from the literature, discussions with a physician treating patients with $\mathrm{PNH}$, and through collaborative efforts among co-authors. The initial draft of the PNH-SQ assessed the presence/absence of 12 symptoms: fatigue, shortness of breath, muscle weakness, headache, abdominal pain, leg/back pain, chest discomfort, sexual difficulties, difficulty sleeping, cognitive problems (i.e., difficulty focusing and difficulty thinking clearly), and difficulty swallowing. If a symptom was reported as present, the participant was then asked to rate symptom severity. The PNH-SQ is intended to be administered daily and has a $24-\mathrm{h}$ recall period.

\section{Participant recruitment}

Interviews with $\mathrm{PNH}$ patients were conducted to (1) identify and confirm the important and relevant symptoms from the patient's perspective (i.e., CE interviews) and (2) to evaluate the respondent's ability to understand and complete the PNH-SQ (i.e., CD).

To this end, we sought to interview 15 patients with $\mathrm{PNH}$. Given that PNH is a rare disease, making recruitment challenging, a conservative estimate of when concept saturation may be reached was set at 15 participants. Following institutional review board approval, potentially eligible study participants were identified through 2 patient groups in the United States: the Aplastic Anemia and Myelodysplastic Syndromes International Foundation (AA-MDS) and the Rare Patient Voice (RPV). Prior to confirming eligibility, patients were presented with study information and provided written informed consent. To be eligible for the study, patients had to meet the following inclusion criteria: at least 18 years of age, have a clinical diagnosis of PNH (written confirmation by the patient's physician was sought), not have an active systemic autoimmune disease, not be currently participating in a clinical trial, and not have an active, co-existing chronic anemia unrelated to PNH (e.g., aplastic anemia or myelodysplastic syndrome).

\section{Patient interviews}

Interviews, approximately $60 \mathrm{~min}$ in duration, were conducted via telephone or in-person. Interview mode was determined by what was most convenient for the participant. Using a semi-structured interview guide, the interviews consisted of 2 distinct parts: (1) CE, to assess the comprehensiveness of symptoms captured by the PNH-SQ and (2) CD, to evaluate the participant's ability to understand and respond to the PNH-SQ. During the CE portion of the interview, participants were asked open-ended questions about their symptoms and experience living with $\mathrm{PNH}$, providing a full, participant-led picture of symptoms and impacts of $\mathrm{PNH}$. To ensure the symptoms discussed by the participant were believed to be related to disease and not a treatment side effect or other health issue, participants were specifically asked about the symptoms they experienced due to their $\mathrm{PNH}$. To ensure that all relevant symptoms were captured in the PNH-SQ, interviewers were instructed to ask the participant if they experienced symptoms from the PNH-SQ that were not mentioned spontaneously. Concept saturation was assessed by dividing interviews into 3 equal groups of 5 participants based on the order in which the interviews were conducted. Saturation is considered to be achieved if a downward trend is observed in the elicitation of new symptoms (i.e., if few or no new symptoms emerge in the final transcript group). Additionally, the concepts mentioned spontaneously by participants were used to confirm the relevance of the patient-centric conceptual model of PNH (Fig. 2). This model shows the symptoms and impacts patients experience and believe are directly related to their $\mathrm{PNH}$, first gathered by review of qualitative literature $[7,8,11-13]$ and then confirmed in the interviews with patients. The conceptual model focuses on disease symptoms and impacts while excluding treatment side effects and related impacts.

During the $\mathrm{CD}$ portion of the interview, participants were asked to complete the PNH-SQ. Participants were instructed to use a "think aloud" procedure in which they verbalize their thoughts as they complete the questionnaire. For example, when participants selected a response for symptom severity, they were asked to describe why they selected that response. Data from CD were used to evaluate the relevance of each item and assess participant's understanding of all instructions, items, recall period, and response options and to confirm the suitability of the recall period and response options. We 


\begin{tabular}{|c|c|c|}
\hline PNH Symptoms & & \\
\hline Fatigue & \multicolumn{2}{|c|}{ Impacts of PNH } \\
\hline Abdominal pain & \multirow{2}{*}{$\begin{array}{l}\text { Impacts on illness } \\
\text { perceptions }\end{array}$} & Preoccupation with disease \\
\hline Headaches & & \multirow{2}{*}{$\begin{array}{l}\text { E.g., thoughts center around disease, } \\
\text { disease center of life }\end{array}$} \\
\hline Shortness of breath & \multirow{2}{*}{$\begin{array}{l}\text { E.g., losing control of own } \\
\text { body, visible symptoms } \\
\text { remind me I'm sick }\end{array}$} & \\
\hline Difficulty swallowing & & Emotional impacts \\
\hline Erectile dysfunction & & \multirow{2}{*}{$\begin{array}{l}\text { E.g., fear, loss of lightheartedness, } \\
\text { stress, helplessness }\end{array}$} \\
\hline Hemoglobinuria & Impact on work/school life & \\
\hline \multirow{2}{*}{$\begin{array}{l}\text { Cardiovascular symptoms (e.g., } \\
\text { chest pain, irregular heartbeat) }\end{array}$} & \multirow{2}{*}{$\begin{array}{l}\text { E.g., problems with work. } \\
\text { no flexibility in planning } \\
\text { studies }\end{array}$} & Physical impacts \\
\hline & & E.g., trouble walking, jogging, standing \\
\hline \multirow{3}{*}{$\begin{array}{l}\text { Cognitive symptoms (e.g., } \\
\text { confusion, poor concentration, } \\
\text { dizziness) }\end{array}$} & \multirow{5}{*}{$\begin{array}{l}\text { Overall quality of life } \\
\text { E.g., normal rhythm of life } \\
\text { affected, missing something } \\
\text { in life, future prospects } \\
\text { limited }\end{array}$} & Impacts on ADLs \\
\hline & & \multirow{2}{*}{$\begin{array}{l}\text { E.g., problems getting household work } \\
\text { done }\end{array}$} \\
\hline & & \\
\hline Bruising/bleeding & & Impacts on relationship \\
\hline Back pain & & E.g., support from family/friends \\
\hline Leg pain & & \\
\hline
\end{tabular}

Fig. 2 Conceptual model of PNH. PNH, paroxysmal nocturnal hemoglobinuria

included 2 items for the cognitive issues concept (i.e., "difficulty thinking clearly" and "difficulty focusing") with the intent of asking patients if they represented the same concept, and, if so, which item best captured their experience. Following the review of the PNH-SQ with the participant, interviewers also asked patients if they thought any symptoms were missing from the PNH-SQ, and if so, which symptoms they would recommend for inclusion.

\section{Interview analysis}

All interviews were audio-recorded, transcribed, coded, and analyzed using NVivo v12.0. For the CE data, the frequency with which participants reported each symptom concept was tallied. For the CD data, the frequency with which participants reported issues with, or understanding of, item content was tallied. Results were summarized in tabular format.

\section{Results}

Most interviews (9/15) were conducted via telephone, 2 were conducted using telephone with video, and 4 were conducted in-person. The mean age of patients was 42.8 years (SD: 10.4), 53.3\% were women, $93.3 \%$ were White, average years since diagnosis was 13.4 years (SD: 10.3 ), and $66.7 \%, 13.3 \%$, and $20.0 \%$ reported that they had very mild/mild, moderate, and severe/ very severe disease, respectively (Table 1 ). Among the 12 patients who were asked about treatment, 7 participants were currently treated with eculizumab. Ultimately, clinical confirmation of a PNH diagnosis was received for 4 participants.

\section{Concept elicitation}

During the interviews, participants mentioned 27 signs or symptoms of PNH either spontaneously or after being probed. Fourteen of 15 (93.3\%) participants reported experiencing symptoms due to $\mathrm{PNH} ; 1$ female participant reported that she did not experience symptoms in the 18 years since her PNH diagnosis. The most common symptoms mentioned were fatigue $(n=13$, all spontaneous), abdominal pain ( $\mathrm{n}=9$, all spontaneous), difficulty swallowing ( $\mathrm{n}=7,4$ spontaneous), sexual difficulties $(n=6,2$ spontaneous), and back pain $(n=6,5$ spontaneous) (Table 2). Symptoms spontaneously mentioned by at least 5 participants included: fatigue $(\mathrm{n}=13)$, abdominal pain $(n=9)$, muscle weakness $(n=5)$, back pain $(n=5)$, cognitive difficulties $(\mathrm{n}=5)$, and shortness of breath $(\mathrm{n}=5)$. In addition to PNH symptoms, participants also frequently spontaneously mentioned signs of $\mathrm{PNH}$, such as dark urine $(\mathrm{n}=11)$, bruising $(\mathrm{n}=4)$, paleness $(\mathrm{n}=3)$, and yellow eyes $(n=1)$. When participants were asked which symptom of PNH was most bothersome, the majority $(n=8)$ considered fatigue to be the most bothersome symptom, followed by cognitive issues $(\mathrm{n}=3)$, and stomach pain $(\mathrm{n}=2)$.

Of the 27 signs and symptoms that were elicited spontaneously or after probing, $66.7 \%$ emerged in the first 5 interviews (Table 3). Two concepts, nausea and 
Table 1 Characteristics of interview participants $(N=15)$

\begin{tabular}{|c|c|c|c|c|}
\hline Participant characteristic & Very mild to mild $(N=10)$ & Moderate $(\mathrm{N}=2)$ & Severe to very severe $(\mathrm{N}=3)$ & $\begin{array}{l}\text { Total } \\
(\mathrm{N}=15)\end{array}$ \\
\hline \multicolumn{5}{|l|}{ Age, years } \\
\hline Mean (SD) & $41.5(11.10)$ & $52.5(3.54)$ & $40.7(9.50)$ & $42.8(10.42)$ \\
\hline Q1 & 33.0 & 50.0 & 31.0 & 33.0 \\
\hline Median & 41.0 & 52.5 & 41.0 & 41.0 \\
\hline Q3 & 47.0 & 55.0 & 50.0 & 50.0 \\
\hline Min-Max & $28-63$ & $50-55$ & $31-50$ & $28-63$ \\
\hline \multicolumn{5}{|l|}{ Sex, n (\%) } \\
\hline Female & $5(50.0)$ & $1(50.0)$ & $2(66.7)$ & $8(53.3)$ \\
\hline Male & $5(50.0)$ & $1(50.0)$ & $1(33.3)$ & $7(46.7)$ \\
\hline \multicolumn{5}{|l|}{ Race, n (\%) } \\
\hline White & $10(100.0)$ & $2(100.0)$ & $2(66.7)$ & $14(93.3)$ \\
\hline Other & $0(0.0)$ & $0(0.0)$ & $1(33.3)$ & $1(6.7)$ \\
\hline \multicolumn{5}{|l|}{ Ethnicity, n (\%) } \\
\hline Hispanic/Latino & $0(0.0)$ & $0(0.0)$ & $1(33.3)$ & $1(6.7)$ \\
\hline Not Hispanic/Latino & $10(100.0)$ & $2(100.0)$ & $2(66.7)$ & $14(93.3)$ \\
\hline \multicolumn{5}{|l|}{ Education, n (\%) } \\
\hline High school diploma (or GED) & $0(0.0)$ & $0(0.0)$ & $2(66.7)$ & $2(13.3)$ \\
\hline Some college or certification program & $1(10.0)$ & $0(0.0)$ & $0(0.0)$ & $1(6.7)$ \\
\hline College or university degree & $5(50.0)$ & $2(100.0)$ & $1(33.3)$ & $8(53.3)$ \\
\hline Graduate degree & $4(40.0)$ & $0(0.0)$ & $0(0.0)$ & $4(26.7)$ \\
\hline \multicolumn{5}{|l|}{ Employment status, n (\%) } \\
\hline Other & $0(0.0)$ & $0(0.0)$ & $1(33.3)$ & $1(6.7)$ \\
\hline Employed full-time & $8(80.0)$ & $0(0.0)$ & $1(33.3)$ & $9(60.0)$ \\
\hline Employed part-time & $1(10.0)$ & $1(50.0)$ & $0(0.0)$ & $2(13.3)$ \\
\hline Homemaker & $0(0.0)$ & $0(0.0)$ & $1(33.3)$ & $1(6.7)$ \\
\hline Retired & $1(10.0 \%)$ & $1(50.0 \%)$ & $0(0.0 \%)$ & $2(13.3 \%)$ \\
\hline \multicolumn{5}{|l|}{ Time since diagnosis, years } \\
\hline Mean (SD) & $11.6(9.11)$ & $27.0(1.41)$ & $10.3(12.10)$ & $13.4(10.25)$ \\
\hline Q1 & 4.0 & 26.0 & 1.0 & 4.0 \\
\hline Median & 10.5 & 27.0 & 6.0 & 12.0 \\
\hline Q3 & 18.0 & 28.0 & 24.0 & 24.0 \\
\hline Min-Max & $0-28$ & $26-28$ & $1-24$ & $0-28$ \\
\hline \multicolumn{5}{|l|}{ Treatment status, n (\%) } \\
\hline Unknown & $3(30.0)$ & $0(0.0)$ & $0(0.0)$ & $3(20.0)$ \\
\hline On treatment & $3(30.0)$ & $2(100.0)$ & $2(66.7)$ & $7(46.7)$ \\
\hline Treatment naïve & $4(40.0)$ & $0(0.0)$ & $1(33.3)$ & $5(33.3)$ \\
\hline
\end{tabular}

GED general educational development, $Q$ quarter, $S D$ standard deviation

ascites, emerged in the last set of interviews but were considered by a physician who treats patients with PNH (co-author J.S.) as idiosyncratic to that patient. As such, concept saturation was reached for PNH symptoms with 15 interviews and the conduct of additional interviews was considered unnecessary. Notably, all 12 symptoms included in the first draft of the PNH-SQ were spontaneously mentioned by at least 1 participant during CE (Table 3). The data from CE were also used to finalize the patient-centric conceptual model of PNH symptoms and impacts (Fig. 2), for which no changes were needed.

\section{Cognitive debrief of the PNH-SQ Item relevance}

Participants completed the PNH-SQ and were then asked about the relevance of each item, regardless of whether they had experienced it in the last $24 \mathrm{~h}$. The majority of study participants found all items in the PNH-SQ relevant (Table 4). Item relevance ranged from $54.5 \%$ (for 
Table 2 Signs and symptoms reported during concept elicitation ( $N=15)$

\begin{tabular}{|c|c|c|c|c|}
\hline Symptoms and signs reported & Spontaneous & Probed & Total N & $\begin{array}{l}\text { Percent } \\
\text { experienced } \\
\text { symptom, \% }\end{array}$ \\
\hline Fatigue ${ }^{*}$ & 13 & 0 & 13 & 87 \\
\hline Dark urine & 11 & 0 & 11 & 73 \\
\hline Abdominal (stomach) pain* & 9 & 0 & 9 & 60 \\
\hline Difficulties swallowing* & 4 & 3 & 7 & 47 \\
\hline Sexual difficulties* & 2 & 4 & 6 & 40 \\
\hline Back pain* & 5 & 1 & 6 & 40 \\
\hline Cognitive difficulties* & 5 & 0 & 5 & 33 \\
\hline Headache* & 4 & 1 & 5 & 33 \\
\hline Muscle weakness* & 5 & 0 & 5 & 33 \\
\hline Shortness of breath* & 5 & 0 & 5 & 33 \\
\hline Bruising & 4 & 0 & 4 & 27 \\
\hline Sleep problems* & 3 & 1 & 4 & 27 \\
\hline Paleness & 3 & 0 & 3 & 20 \\
\hline Leg pain* & 3 & 0 & 3 & 20 \\
\hline Chest discomfort* & 2 & 0 & 2 & 13 \\
\hline Dizziness & 2 & 0 & 2 & 13 \\
\hline Fast heart rate & 2 & 0 & 2 & 13 \\
\hline Muscle aches & 2 & 0 & 2 & 13 \\
\hline Yellow eyes & 1 & 0 & 1 & 7 \\
\hline Sore spleen & 1 & 0 & 1 & 7 \\
\hline Sensitive nerves & 1 & 0 & 1 & 7 \\
\hline Heartburn & 1 & 0 & 1 & 7 \\
\hline Hoarse voice & 1 & 0 & 1 & 7 \\
\hline Vision problems & 1 & 0 & 1 & 7 \\
\hline Urinary spasms & 1 & 0 & 1 & 7 \\
\hline Ascites & 1 & 0 & 1 & 7 \\
\hline Nausea & 0 & 1 & 1 & 7 \\
\hline
\end{tabular}

* Denotes an item listed in the Paroxysmal Nocturnal Hemoglobinuria-Symptom Questionnaire (PNH-SQ)

"difficulty sleeping") to $100 \%$ (for "fatigue"). All symptoms on the PNH-SQ had been experienced by at least 1 patient in the last $24 \mathrm{~h}$. At least one-third of participants reported experiencing fatigue $(n=7)$, headache $(n=5)$, or cognitive difficulties $(n=5)$, in the last $24 \mathrm{~h}$ (Fig. 3). There appeared to be some variability in how often patients experienced symptoms. For example, while $60 \%$ of patients $(9 / 15)$ experienced abdominal pain due to their PNH in the past, only 1 participant reported having abdominal pain in the past $24 \mathrm{~h}$. In contrast, $>50 \%$ of patients reporting having ever experienced fatigue, back pain, headache, cognitive difficulties, muscle weakness, shortness of breath, or difficulty sleeping also reported having experienced the symptom in the past $24 \mathrm{~h}$.

While an almost equal number of participants felt that both "difficulty focusing" (10/13) and "thinking clearly" (10/12) were relevant, most patients $(9 / 13)$ believed that the items were interchangeable and should be combined into 1 item. Patients tended to describe "difficulty focusing" in narrow terms relating to a specific task and described "difficulty thinking clearly" in broader concepts which included confusion, memory, or language retrieval issues.

\section{Understanding of PNH-SQ}

Participant understanding of instructions and symptom concepts was high, between $>90$ and $100 \%$ demonstrated understanding. Participant understanding of the response options was very high for each item, from $>80$ to $100 \%$. Further, $>80-100 \%$ stated that they would be able to easily recall the symptom and its severity within the last $24 \mathrm{~h}$.

\section{PNH-SQ refinement and finalization}

Since participants felt that "difficulty focusing" was interchangeable with "difficulty thinking clearly," we decided 
Table 3 Concept saturation analysis

\begin{tabular}{|c|c|c|c|c|}
\hline Symptom & Set $1 n=5$ & Set $2 n=5$ & Set $3 n=5$ & $\begin{array}{l}\text { Saturation } \\
\text { met? }\end{array}$ \\
\hline Fatigue & $\checkmark$ & $\checkmark$ & $\checkmark$ & Y \\
\hline Sleep problems & $\checkmark$ & $\mathbf{x}$ & $\checkmark$ & Y \\
\hline Abdominal pain & $\checkmark$ & $\checkmark$ & $\checkmark$ & Y \\
\hline Chest discomfort & $\checkmark$ & $x$ & $\checkmark$ & Y \\
\hline Shortness of breath & $\checkmark$ & $\checkmark$ & $\checkmark$ & Y \\
\hline Muscle weakness & $x$ & $\boldsymbol{J}^{*}$ & $\checkmark$ & Y \\
\hline $\begin{array}{l}\text { Cognitive difficul- } \\
\text { ties }\end{array}$ & $\checkmark$ & $\checkmark$ & $\checkmark$ & Y \\
\hline $\begin{array}{l}\text { Difficulties swal- } \\
\text { lowing }\end{array}$ & $\checkmark$ & $\checkmark$ & $x$ & Y \\
\hline Sexual difficulties & $x$ & $J^{*}$ & $x$ & Y \\
\hline Yellow eyes & $\checkmark$ & $\mathbf{x}$ & $x$ & Y \\
\hline Headache & $\checkmark$ & $\checkmark$ & $x$ & Y \\
\hline Sore spleen & $\checkmark$ & $x$ & $x$ & Y \\
\hline Sensitive nerves & $\checkmark$ & $x$ & $x$ & Y \\
\hline Back pain & $\checkmark$ & $\checkmark$ & $\checkmark$ & Y \\
\hline Dark urine & $\checkmark$ & $\checkmark$ & $\checkmark$ & Y \\
\hline Paleness & $\checkmark$ & $x$ & $\checkmark$ & Y \\
\hline Bruising & $\checkmark$ & $\checkmark$ & $\checkmark$ & Y \\
\hline Heartburn & $\checkmark$ & $x$ & $x$ & Y \\
\hline Hoarse voice & $x$ & $\boldsymbol{J}^{*}$ & $x$ & Y \\
\hline Fast heart rate & $x$ & $\boldsymbol{J}^{*}$ & $\checkmark$ & Y \\
\hline Muscle aches & $x$ & $\boldsymbol{J}^{*}$ & $\checkmark$ & Y \\
\hline Urinary spasms & $\checkmark$ & $x$ & $x$ & Y \\
\hline Ascites & $x$ & $x$ & $\boldsymbol{J}^{*}$ & $\mathrm{~N}$ \\
\hline
\end{tabular}

$\checkmark$ Concept discussed in interview set

$\mathbf{x}$ Concept not discussed in interviewset $N$ No, $Y$ Yes

${ }^{*}$ Represents emergence of a new concept to retain only 1 item. Given that the descriptions provided by participants for "difficulty thinking clearly" aligned more closely with the broader concept of cognitive difficulties and "brain fog," that item was retained.

The item "sexual difficulties" was also removed. Sexual difficulties among men were primarily experienced as erectile dysfunction (ED) while 1 woman experienced sexual difficulties as low sex drive. While 6 of the 15 participants ( 1 female, 5 males) reported having experienced sexual difficulties, 13 participants had not experienced this symptom in the past $24 \mathrm{~h}$ (Fig. 3). Further, the frequency with which patients experienced sexual difficulty varied. Two male participants reported experiencing ED on an ongoing basis, 1 within the last $24 \mathrm{~h}$ and 1 within 5 days of completing the PNH-SQ with the 3 other male participants experiencing ED only in the past (a single instance of ED 2 and 9 years prior for 2 participants). The woman reported experiencing a low sex drive on a weekly basis. In addition, 1 male participant reported that they received clinical confirmation that their ED was not attributable to $\mathrm{PNH}$ and the woman reported that the low sex drive was due to her anemia. Due to the limited relevance of the item to women with PNH (1/8), the variable frequency with which it occurred, and the fact that the experience of the symptom could be due to factors external to $\mathrm{PNH}$, the item was dropped. The response options were not modified from the original version as they were well understood and considered appropriate for each item.

Lastly, several minor changes were made to facilitate the e-administration of the measure. The final instrument included questions on 10 symptoms of $\mathrm{PNH}$ : abdominal pain, chest discomfort, difficulty sleeping, difficulty swallowing, difficulty thinking clearly, fatigue, headache,

Table 4 Relevancy of items of the PNH-SQ

\begin{tabular}{|c|c|c|c|c|c|}
\hline & Relevant & Not relevant & Maybe relevant & $\begin{array}{l}\text { Number of participants } \\
\text { asked }\end{array}$ & $\begin{array}{l}\text { Percent } \\
\text { relevant, } \\
\%\end{array}$ \\
\hline Fatigue & 15 & 0 & 0 & 15 & 100 \\
\hline Shortness of breath & 8 & 1 & 4 & 13 & 62 \\
\hline Muscle weakness & 9 & 3 & 1 & 13 & 69 \\
\hline Headache & 8 & 4 & 0 & 12 & 67 \\
\hline Abdominal pain & 10 & 2 & 0 & 12 & 83 \\
\hline Pain in back or legs & 11 & 1 & 0 & 12 & 92 \\
\hline Chest discomfort & 8 & 5 & 0 & 13 & 62 \\
\hline Sexual difficulties & 9 & 0 & 2 & 11 & 82 \\
\hline Difficulty sleeping & 6 & 1 & 4 & 11 & 55 \\
\hline Difficulty focusing & 11 & 1 & 1 & 13 & 85 \\
\hline Difficulty thinking clearly & 10 & 2 & 0 & 12 & 83 \\
\hline Difficulty swallowing & 10 & 3 & 0 & 13 & 77 \\
\hline
\end{tabular}




\section{Comparison of symptoms ever experienced vs. experienced in the last 24 hours}

Experienced in last 24 hours

Ever experienced

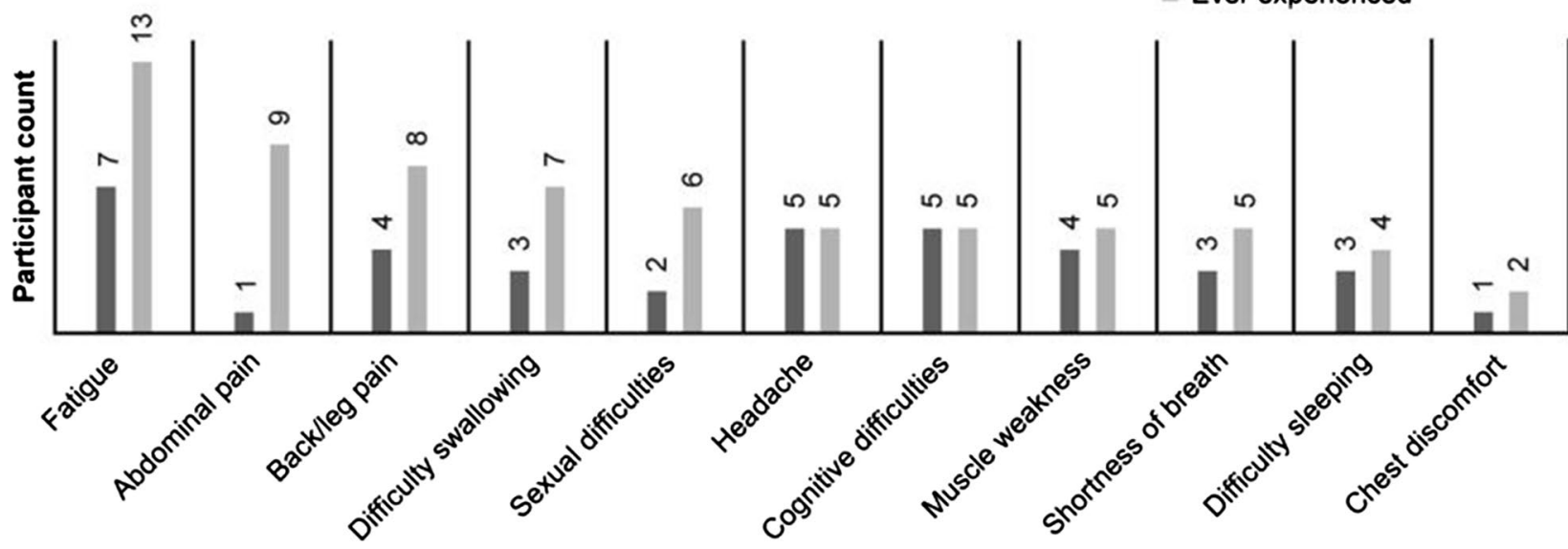

Symptom

Fig. 3 Frequency of PNH symptoms experienced. PNH paroxysmal nocturnal hemoglobinuria

muscle weakness, pain in the legs or back, and shortness of breath. The PNH-SQ, intended for daily e-administration, asks the patient to report on the presence/absence of each symptom over a 24 -h period and to rate the severity of each symptom they have experienced.

\section{Discussion}

This study describes the development of the PNH-SQ, a content-valid questionnaire developed in accordance with regulatory guidelines [9] and scientific best practices [14]. A literature review of instruments used to assess $\mathrm{PNH}$ symptoms in a clinical trial context informed the need for the development of a daily symptom diary that assesses the most salient symptoms of PNH from the perspective of the patient. The PNH-SQ was developed by combining the findings from the literature review, as well as expert clinician advice, and was further refined through patient interviews.

CE interviews with $15 \mathrm{PNH}$ patients confirmed that most patients (i.e., $>90 \%$ ) experience symptoms due to their $\mathrm{PNH}$ and that they experience a wide variety of symptoms [7, 8, 13]. Even while many (7/15) participants were receiving treatment for their $\mathrm{PNH}$, study participants reported experiencing over 27 signs and symptoms due to $\mathrm{PNH}$ with the most common being fatigue (87\%), abdominal pain (60\%), dysphagia (47\%), sexual difficulties (40\%), and back pain (40\%); and onethird (33\%) of patients reported cognitive difficulties, headache, muscle weakness, and shortness of breath. A small qualitative study of 29 patients by Weitz et al. [7] reported similarly high rates of fatigue $(97 \%)$ abdominal pain (59\%), dysphagia (41\%), and sexual difficulties (47\%) [8]. Likewise, similar rates of erectile dysfunction (38\%) and fatigue $(80 \%)$ were reported in the International PNH registry. However, both in the International PNH Registry and in the Weitz study, patients reported substantially higher rates of headache $(33 \%$ vs. $63 \%$ and $76 \%$, respectively) and shortness of breath (33\% vs. $64 \%$ and $66 \%$, respectively) $[7,8]$. Further, participants in our study also reported experiencing leg/back pain (53\%), muscle weakness (33\%), and sleep problems (27\%), which were not reported by the patients in the International PNH Registry or in the qualitative study [7, 8]. Differences in the experience of symptoms may be driven by case mix, including severity of PNH (most patients in our study had self-reported mild disease but severity was not reported in the registry [7] or Weitz study [8]) and treatment status (none of the patients in the Weitz study [8] were treated with eculizumab vs. $25 \%$ in the registry [7] and $>45 \%$ in our study).

The significance of fatigue in the PNH experience is underscored by the fact that fatigue was the most commonly experienced symptom $(13 / 15 ; 86.7 \%)$ and was considered the most bothersome symptom by the majority $(8 / 15)$ of patients in this study. The proportion of individuals with $\mathrm{PNH}$ experiencing fatigue corresponds with findings from the International PNH Registry and the Weitz study; both of which found that fatigue was the most frequently reported symptom (80\% and $97 \%$, respectively) [8]. Moreover, in our study, nearly one-half 
of participants experienced fatigue in the prior 24-h (7/15) and more than half (4/7) rated the severity as moderate to severe. This is consistent with a cross-sectional survey including 74 participants with $\mathrm{PNH}$ in which mean levels of fatigue were severe [15]. Our findings are also consistent with the Weitz study, in which fatigue was reported to occur frequently or almost constantly in $52 \%$ of the study participants. Overall, the evidence from this and other studies demonstrate that fatigue is a frequently experienced symptom of PNH.

Fatigue has been positioned as a key secondary endpoint in 2 phase-3 PNH clinical trials [16, 17], both using the FACIT-Fatigue to measure the symptom. Although, the FACIT-Fatigue has been shown to comprehensively capture the fatigue that $\mathrm{PNH}$ patients with experience [8], it uses a 7-day recall period. As previously discussed, most patients with $\mathrm{PNH}$ experience fatigue frequently and the use of a 7-day recall period does not allow for the measurement of day-to-day variability in fatigue that participants may experience due to changes in disease activity or as a result of the treatment cycle. Although we acknowledge that there is some support for longer recall periods [18], the FDA's PRO guidance suggests that a shorter recall period is preferable for instruments used in clinical trials due to concerns about recall bias [10]. As has been demonstrated in other chronic diseases, using a 7-day recall period may introduce a recall bias with patients recalling higher symptom intensity than when a daily recall period is used [19, 20]. For example, a study examining fatigue among 97 rheumatology patients confirmed that recall bias was not significant when comparing daily recalls of fatigue to momentary ratings, suggesting that a 24-recall period is suitable for fatigue [21]. The use of a daily symptom questionnaire, such as the PNH-SQ, which can capture day-to-day changes in fatigue and other symptoms can provide a more nuanced representation of the patient's experience of these symptoms. Additionally, in measuring the daily variability in fatigue, the PNH-SQ may be a more sensitive measure than the FACIT-Fatigue in detecting changes in this symptom.

A widely used PRO measure in PNH clinical trials [16, 17], the EORTC QLQ-C30 was found to inadequately capture symptoms experienced by $\mathrm{PNH}$ patients [8]. The study found that while the measure was clear and easy to understand, it contained several items that were of no or low relevance to US patients with $\mathrm{PNH}$, including items such as vomiting, needing help with eating and dressing, nausea, and diarrhea. In our study, none of these signs or symptoms emerged as concepts important to patients with PNH. Importantly, the most common symptoms of $\mathrm{PNH}$ such as headache, dysphagia, and abdominal pain reported by participants in that study and in our study are not directly assessed by the EORTC QLQ-C30. In addition, the most commonly experienced and bothersome symptom of $\mathrm{PNH}$, fatigue, is not directly assessed in the EORTC-QLQ-30, which includes a question about "tiredness" rather than fatigue. In fact, the conclusion from the study evaluating the EORTC QLQ-30 as a PRO for patients with PNH was to add items regarding common symptoms of $\mathrm{PNH}$, namely, abdominal pain, headache, and shortness of breath [8]. Evidence from qualitative studies, including our own, suggests that the EORTC QLQ-C30 does not assess all symptoms that are meaningful to patients with $\mathrm{PNH}$ [8], an aspect that is critical in the use of PROs in clinical trials [10].

In contrast, to ensure that the items of the PNH-SQ were relevant and comprehensively captured, participants in our study were asked to comment on the relevance of each item. Item relevance on the PNH-SQ was $>50 \%$ for all items (range 54.5-100\%). Furthermore, when asked about measure comprehensiveness, no patient suggested adding further symptoms. These findings support the content validity and comprehensiveness of the PNH-SQ.

A new PRO instrument-the Quality of Life Questionnaire for patients with Aplastic Anemia and/or Paroxysmal Nocturnal Hemoglobinuria (QLQ-AA/PNH) - was recently developed to measure quality of life in patients with aplastic anemia and $\mathrm{PNH}[6,22]$. The instrument was developed with significant patient and clinician input, has 54 items with a 14-day recall period for most items and a 6-month recall period for 2 items. While the measure does assess some symptoms, the focus of the items are quality of life impacts and healthcare experience [22]. Indeed, the instrument addresses a significant need for a quality of life tool specific to patients with $\mathrm{PNH}$ but it does not comprehensively assess $\mathrm{PNH}$ symptoms, their frequency, or their severity. Further, as previously discussed, there is day-to-day variation in experience of $\mathrm{PNH}$ symptoms and the 2-week recall period may introduce recall bias in the measurement of symptoms [19-21]. The QLQ-AA/PNH may be a complementary instrument to the PNH-SQ; together these instruments can comprehensively assess both symptom burden and quality of life impact of PNH.

Recently, the patient-reported outcome questionnaire for aplastic anemia and paroxysmal nocturnal hemoglobinuria (PRO-AA/PNH) was developed [23]. However, upon comparison of the measures, only some of the 10 PNH-SQ items are also measured in the PRO AA/PNH (e.g., fatigue, shortness of breath, difficulty concentrating, and dysphagia). For instance, the PRO AA/PNH captures pain in one general question, whilst the $\mathrm{PNH}$ SQ asks about specific issues with pain because this was a significant area of concern raised by patients (items 
include back, leg, and stomach pain). Additionally, the PRO-PNH/AA captures some signs of PNH (eg, hemoglobinuria, jaundice) and impacts (mood, trouble doing strenuous activities). The PNH-SQ focuses primarily on symptoms of $\mathrm{PNH}$ rather than signs or impacts of $\mathrm{PNH}$, and was developed to capture the most relevant symptoms that only patients discussed (e.g., erectile dysfunction was not included because on a day-to-day basis, this was not reported as an issue). Thus, the PNH-SQ may be a more sensitive measure of change in symptoms. Furthermore, the impact of PNH is multi-faceted and arguably warrants a more comprehensive approach to assessment by using something like the QLQ-AA/PNH [6].

The findings from this study must be understood in the context of several limitations. The study included 15 patients with $\mathrm{PNH}$, all of whom were from the US and all interviews were conducted in English which may impact its cross-cultural validity. However, a translatability assessment was conducted to ensure that the instructions, items, and response options could be translated across a variety of different languages, as individuals who speak different languages may express their symptom experience differently. Moreover, in order to obtain a diverse group of participants and best capture the wide range of $\mathrm{PNH}$ experiences, recruitment targets were set by age group, ethnicity, race, educational attainment, treatment status, and self-reported disease severity. While there is no evidence to suggest that PNH symptom experience would differ across race or ethnicity, no participants in this study were African American and only 1 Hispanic individual participated in the study. Nevertheless, since saturation was reached, the conduct of additional interviews is unlikely to result in the elicitation of additional, key symptoms that are relevant to patients with $\mathrm{PNH}$.

Further, clinical confirmation of PNH diagnosis was only possible for 4 patients and thus the study relied primarily on self-reported PNH diagnosis for most participants. Considering the nature of the study, the detailed disease-related questions, and the fact that participants were recruited from a $\mathrm{PNH}$ or rare disease advocacy organization, it is unlikely that those participants who self-reported their PNH diagnosis did not in fact have $\mathrm{PNH}$. Furthermore, PNH patients with AA were not included in this study; this measure may therefore not comprehensively capture the experience of patients who have both diseases.

In conclusion, the PNH-SQ is a new, content-valid PRO measure suitable for assessing daily symptoms experienced by patients with $\mathrm{PNH}$ in a clinical trial context. The development of the PNH-SQ was informed by the literature and by clinician and patient input. While further research is necessary to determine the psychometric properties of the PNH-SQ (i.e., reliability, construct validity, ability to detect change, meaningful change threshold, and scoring algorithm), this study demonstrates that the PNH-SQ is a clear and easy to understand questionnaire that has comprehensive coverage of relevant PNH symptoms. Lastly, because the PNHSQ was developed for daily administration, it can capture daily variations in symptoms of $\mathrm{PNH}$ patients, providing a more granular assessment of symptom changes among patients enrolled in clinical trials.

\section{Abbreviations \\ AA-MDS: Aplastic Anemia and Myedolysplastic Syndromes International FOUNDATION; CD: Cognitive debriefing; CE: Concept elicitation; ED: Erectile dysfunction; EORTC QLQ-C30: European Organisation for Research and Treat- ment of Cancer Quality of Life of Cancer Patients; FACIT-Fatigue: Functional assessment of chronic illness therapy-fatigue; FDA: Food and drug administra- tion; PNH: Paroxysmal nocturnal hemoglobinuria; PNH-SQ: PNH symptom questionnaire; PRO: Patient-reported outcomes; PRO-AA/PNH: Patient- reported outcome questionnaire aplastic anemia and paroxysmal noctur- nal hemoglobinuria; RPV: Rare patient voice; QLQ-AA/PNH: Quality of life questionnaire for patients with aplastic anemia and/or paroxysmal nocturnal hemoglobinuria.}

\section{Acknowledgements}

We would like to thank the patients for their involvement in this study. We are grateful to the Aplastic Anemia and MDS International Foundation (AA-MDS) as well as the Rare Patient Voice (RPV) who were vital in connecting researchers to individuals with PNH. Additionally, we would like to thank Dr. Anita Hill for providing input as to which symptoms to include in the first draft of the questionnaire

\section{Authors' contributions}

TS and JJ planned and designed the study. SK and RPD contributed to the data collection and analysis of the results; RPD, JJ, SK, TS and JS contributed to the manuscript and its revisions. All authors read and approved the final manuscript.

Funding

This study was sponsored by Regeneron.

\section{Availability of data and materials}

The datasets used and analyzed during the current study are available from the corresponding author on reasonable request.

\section{Declarations}

Ethics approval and consent to participate

This study was performed in accordance with the Declaration of Helsinki and was approved by the Copernicus Group IRB, Panel 18 (07-Nov-2020) CGIBR protocol \# 20182896.

\section{Consent for publication}

Not applicable

\section{Competing interests}

R.P.D. was an employee of Clinical Outcomes Solutions; J.J.J. is an employee of Regeneron Pharmaceuticals, Inc.; S.K. and T.S. are employees of Clinical Outcomes Solutions; J.S. has the following disclosures: Regeneron Pharmaceuticals, Inc.: Consultancy; Incyte: Consultancy, Honoraria, Research Funding, Speakers Bureau; CTI Pharma: Research Funding; Onconova: Research Funding; Alexion: Consultancy, Honoraria, Research Funding, Speakers Bureau; Celgene: Consultancy, Honoraria, Research Funding, Speakers Bureau; Apellis: Membership on an entity's Board of Directors or advisory committees; Otsuka: 
Consultancy, Honoraria; Novartis: Consultancy, Honoraria; Astex Pharma: Research Funding; Sanofi: Consultancy, Honoraria, Speakers Bureau.

\section{Author details}

${ }^{1}$ Clinical Outcomes Solutions, Chicago, IL, USA. ${ }^{2}$ Regeneron Pharmaceuticals, Inc., 777 Old Saw Mill River Rd, Tarrytown, NY 10591, USA. ${ }^{3}$ Clinical Outcomes Solutions, Folkestone, UK. ${ }^{4}$ Rush University Medical Center, Chicago, IL, USA.

Received: 27 May 2021 Accepted: 15 September 2021

Published online: 28 September 2021

\section{References}

1. Mastellos DC et al (2018) Expanding complement therapeutics for the treatment of paroxysmal nocturnal hemoglobinuria. Semin Hematol 55(3):167-175

2. Mitchell R et al (2017) Path to diagnosis of paroxysmal nocturnal hemoglobinuria: the results of an exploratory study conducted by the aplastic anemia and MDS international foundation and the national organization for rare disorders utilizing an internet-based survey. SM Clin Med Oncol 1(1):1001

3. Young NS et al (2009) The management of paroxysmal nocturnal hemoglobinuria: recent advances in diagnosis and treatment and new hope for patients. Semin Hematol 46(1 Suppl 1):S1

4. Hill A et al (2006) The incidence and prevalence of paroxysmal nocturnal hemoglobinuria $(\mathrm{PNH})$ and survival of patients in Yorkshire. American Society of Hematology, Washington

5. Jalbert JJ et al (2019) Epidemiology of PNH and real-world treatment patterns following an incident PNH diagnosis in the US. American Society of Hematology, Washington

6. Groth M et al (2013) Development of a disease-specific quality of life questionnaire for patients with aplastic anemia and/or paroxysmal nocturnal hemoglobinuria (QLQ-AA/PNH). American Society of Hematology, Washington

7. Schrezenmeier $\mathrm{H}$ et al (2014) Baseline characteristics and disease burden in patients in the International Paroxysmal Nocturnal Hemoglobinuria Registry. Haematologica 99(5):922-929

8. Weitz I et al (2013) Cross-sectional validation study of patient-reported outcomes in patients with paroxysmal nocturnal haemoglobinuria. Intern Med J 43(3):298-307

9. Committee for Medicinal Products for Human Use (2005) Reflection paper on the regulatory guidance for the use of health-related quality of life $(\mathrm{HRQL})$ measures in the evaluation of medicinal products. European Medicines Agency, London

10. US Department of Health and Human Services FDA Center for Drug Evaluation and Research, US Department of Health and Human Services FDA Center for Biologics Evaluation and Research, and U.S. Department of Health and Human Services FDA Center for Devices and Radiological
Health (2006) Guidance for industry: patient-reported outcome measures: use in medical product development to support labeling claims: draft guidance. Health Qual Life Outcomes 4:79

11. Shammo JM et al (2015) Path to diagnosis of paroxysmal nocturnal hemoglobinuria: the results of an exploratory study conducted by the Aplastic Anemia and Myelodysplastic Syndrome International Foundation and the National Organization for Rare Disorders utilizing an Internet-based survey. American Society of Hematology, Washington

12. Meyers $\mathrm{G}$ et al (2007) Disease-related symptoms reported across a broad population of patients with paroxysmal nocturnal hemoglobinuria. American Society of Hematology, Washington

13. Groth M et al (2017) Development of a disease-specific quality of life questionnaire for patients with aplastic anemia and/or paroxysmal nocturnal hemoglobinuria (QLQ-AA/PNH) - report on phases I and II. Ann Hematol 96(2):171-181

14. Patrick DL et al (2011) Content validity_establishing and reporting the evidence in newly developed patient-reported outcomes (PRO) instruments for medical product evaluation: ISPOR PRO Good Research Practices Task Force report: part 2-assessing respondent understanding. Value Health 14(8):978-988

15. Escalante CP et al (2019) Fatigue, symptom burden, and health-related quality of life in patients with myelodysplastic syndrome, aplastic anemia, and paroxysmal nocturnal hemoglobinuria. Cancer Med 8(2):543-553

16. Kulasekararaj AG et al (2019) Ravulizumab (ALXN1210) vs eculizumab in C5-inhibitor-experienced adult patients with $\mathrm{PNH}$ : the 302 study. Blood 133(6):540-549

17. Hillmen P et al (2006) The complement inhibitor eculizumab in paroxysmal nocturnal hemoglobinuria. N Engl J Med 355(12):1233-1243

18. Cella D, Stone AA (2015) Health-related quality of life measurement in oncology: advances and opportunities. Am Psychol 70(2):175

19. Stull DE et al (2009) Optimal recall periods for patient-reported outcomes: challenges and potential solutions. Curr Med Res Opin 25(4):929-942

20. Broderick JE et al (2008) The accuracy of pain and fatigue items across different reporting periods. Pain 139(1):146-157

21. Schneider S et al (2011) Peak and end effects in patients' daily recall of pain and fatigue: a within-subjects analysis. J Pain 12(2):228-235

22. Niedeggen C et al (2019) Design and development of a disease-specific quality of life tool for patients with aplastic anaemia and/or paroxysmal nocturnal haemoglobinuria (QLQ-AA/PNH) - a report on phase III. Ann Hematol 98(7):1547-1559

23. Weisshaar K et al (2020) Development of a patient-reported outcome questionnaire for aplastic anemia and paroxysmal nocturnal hemoglobinuria (PRO-AA/PNH)

\section{Publisher's Note}

Springer Nature remains neutral with regard to jurisdictional claims in published maps and institutional affiliations.

\section{Submit your manuscript to a SpringerOpen ${ }^{\circ}$ journal and benefit from:}

- Convenient online submission

- Rigorous peer review

- Open access: articles freely available online

- High visibility within the field

- Retaining the copyright to your article

Submit your next manuscript at $\boldsymbol{~ s p r i n g e r o p e n . c o m ~}$ 